\title{
Estratégias governamentais para a garantia do direito humano à alimentação adequada e saudável no enfrentamento à pandemia de Covid-19 no Brasil
}

\author{
Government strategies to ensure the human right to adequate \\ and healthy food facing the Covid-19 pandemic in Brazil
}

Aline do Monte Gurgel (https://orcid.org/0000-0002-5981-3597) ${ }^{1}$

Carla Caroline Silva dos Santos (https://orcid.org/0000-0002-1808-7760) ${ }^{2}$

Kelly Poliany de Souza Alves (https://orcid.org/0000-0001-7293-4628) ${ }^{3}$

Juciany Medeiros de Araujo (https://orcid.org/0000-0001-6205-0116) ${ }^{2}$

Vanessa Sá Leal (https://orcid.org/0000-0001-9492-2580) ${ }^{4}$
${ }^{1}$ Instituto Aggeu Magalhães, Fundação Oswaldo Cruz. Av. Prof. Moraes Rego s/n Cidade Universitária. 50670420 Recife PE Brasil.

alinemgurgel@hotmail.com ${ }^{2}$ Secretaria de Saúde do Recife. Recife PE Brasil. ${ }^{3}$ ACT Promoção da Saúde. Rio de Janeiro RJ Brasil. ${ }^{4}$ Centro Acadêmico de Vitória, Universidade Federal de Pernambuco. Recife PE Brasil.

\begin{abstract}
The Covid-19 pandemic revealed a concrete and immediate threat to food and nutrition security (FNS), especially for vulnerable groups. This study aimed to identify government strategies implemented in Brazil to provide the Human Right to Adequate and Healthy Food in high social vulnerability contexts during the Covid-19 pandemic. A cross-sectional study was carried out, with analysis of official documents published between March 20 and July 30, 2020, by the Federal Government, Federal District, Brazilian states, and capitals, focusing on measures to ensure availability and physical or financial access to food. Strategies implemented mainly involve food distribution and minimum income assurance. The following were implemented: Basic Emergency Income (Federal Government); Food Acquisition Program (PAA), and emergency financial aid (states); emergency food donation programs (states and municipalities). Existing measures were adapted to the pandemic, such as the National School Food Program (PNAE), the National Food Acquisition Program (PAA), and the distribution of food and staple food baskets. While essential, these strategies have limited scope and are insufficient to ensure FNS.

Key words Covid-19, Food and nutrition security, Public policy, Health vulnerability
\end{abstract}

Resumo A pandemia de Covid-19 revelou a existência de ameaça concreta e imediata à segurança alimentar e nutricional (SAN), em especial de grupos vulnerabilizados. O estudo buscou identificar as estratégias governamentais implementadas no Brasil para prover o Direito Humano à Alimentação Adequada e Saudável em contextos de elevada vulnerabilidade social frente à Covid-19. Foi realizado um estudo transversal, com análise de documentos oficiais publicados entre 20 de março e 30 de julho de 2020 pela União, Distrito Federal, estados e capitais brasileiras, com foco em medidas que assegurem disponibilidade e acesso físico ou financeiro a alimentos. As estratégias implementadas envolvem fundamentalmente distribuição de alimentos e garantia de renda mínima. Foram instituídas: Renda Básica Emergencial (União); Programa de Aquisição de Alimentos (PAA) e auxílio financeiro emergencial (estados); programas de doação emergencial de alimentos (estados e municípios). Medidas existentes foram adaptadas frente à pandemia, como o Programa Nacional de Alimentação Escolar (PNAE), o Programa de Aquisição de Alimentos (PAA) nacional, a distribuição de alimentos e de cestas básicas. Embora importantes, essas estratégias têm alcance limitado e são insuficientes para assegurar a SAN. Palavras-chave Covid-19, Segurança alimentare nutricional, Política pública, Vulnerabilidade em saúde 


\section{Introdução}

Em dezembro de 2019 foi identificado um novo coronavírus responsável por uma síndrome respiratória aguda grave que ficou conhecida como Covid-19. A doença espalhou-se rapidamente no mundo e, apesar da adoção de medidas de contenção e isolamento rigorosas em diversas regiões, a Covid-19 foi considerada uma Emergência de Saúde Pública de Importância Internacional e classificada pela Organização Mundial de Saúde (OMS) como pandemia em 11 de março de $2020^{1}$. Mesmo com a significativa subnotificação de casos, ocasionada principalmente pela baixa testagem, em 8 de agosto de 2020 o Brasil registrou 100.000 óbitos pela doença ${ }^{2}$.

A pandemia não afeta de forma equânime os diferentes territórios e populações, tanto em relação à morbimortalidade, quanto às suas repercussões políticas, sociais e econômicas. Seus principais impactos ocorrem em grupos populacionais vulnerabilizados, estando intimamente relacionados a questões de gênero, raça/etnicidade e classe, principalmente nas periferias ${ }^{3,4}$.

Dados da Covid-19 ao redor do mundo revelam uma ameaça concreta e imediata referente à segurança alimentar e nutricional (SAN) de populações mais vulnerabilizadas, particularmente povos e comunidades tradicionais ${ }^{5,6}$. O número de pessoas que sofrem de fome crônica pode aumentar drasticamente, resultando no incremento global da insegurança alimentar e nutricional (InSAN). A garantia das condições de sobrevivência é essencial para o enfrentamento das mudanças forçadas pela pandemia, devendo ser prioridade para os gestores públicos, particularmente aquelas relacionadas à SAN de populações vulneráveis. A situação da Covid-19 pode reintroduzir o Brasil no mapa da fome, desvelando a incapacidade do projeto ultraneoliberal do governo de assegurar direitos e responder adequadamente à crise ${ }^{7}$.

Assim, esse estudo buscou identificar as estratégias governamentais implementadas no Brasil para a garantia do Direito Humano à Alimentação Adequada e Saudável (DHAAS) frente à pandemia de Covid-19, com foco nas medidas que possam contribuir diretamente para a garantia da disponibilidade e do acesso físico ou financeiro aos alimentos, em especial às populações mais vulneráveis.

\section{Metodologia}

Foi realizado um estudo transversal, exploratório e descritivo, a partir da análise documental ${ }^{8}$. Foram analisados documentos oficiais (atos normativos, portarias, resoluções e afins) publicados pela União, Distrito Federal (DF), estados e capitais brasileiras, referentes a estratégias governamentais direcionadas à garantia da disponibilidade e do acesso físico ou financeiro a alimentos, em especial às populações vulneráveis. Também foram consultadas informações disponíveis nos sites oficiais dos governos federal, estaduais e municipais e de órgãos específicos que atuam na gestão de ações e programas relacionados à SAN, como cidadania, assistência social, ação social, defesa social, direitos humanos, agricultura e educação. Foram consideradas publicações realizadas entre 20 de março, quando foi reconhecido o estado de calamidade pública no país em decorrência da pandemia de Covid-19, e 30 de junho de 2020.

Procedeu-se a análise temática do conteúdo dos documentos, seguindo as etapas de pré-análise, análise, tratamento dos resultados, inferência e interpretação, considerando conteúdos explícitos e implícitos nos documentos. Os dados foram organizados segundo estratégia/programa governamental. As informações extraídas dos documentos selecionados foram sistematizadas segundo unidades da federação e capitais, e classificadas de acordo com a finalidade da estratégia e características de sua implementação durante a pandemia.

Quanto à finalidade, as estratégias foram classificadas conforme as dimensões de garantia do DHAAS em: a) disponibilidade de alimentos (ações junto a produtores para facilitar oferta de alimentos adequados e saudáveis); b) acesso físico ou financeiro aos alimentos (ações de distribuição de alimentos ou de renda que contribuam para a compra de alimentos). Quanto à característica de implementação, as estratégias foram organizadas em: a) novas estratégias criadas devido à pandemia; b) estratégias pré-existentes, alteradas devido à emergência sanitária.

\section{Resultados}

No contexto da pandemia, foram identificadas a estruturação de novas iniciativas e a adequação de estratégias já implementadas no país que contribuem para a SAN. As novas medidas governamentais criadas foram: Renda Básica Emergen- 
cial (Governo Federal); Programas de Aquisição de Alimentos (PAA) estaduais e a doação emergencial de alimentos (estados e municípios). As medidas pré-existentes que sofreram alterações devido à pandemia são: Programa Nacional de Alimentação Escolar (PNAE), Programa de Aquisição de Alimentos (PAA) nacional, Programa Bolsa Família (PBF), Benefício de Prestação Continuada (BPC), distribuição de alimentos por meio dos equipamentos públicos de SAN (Quadro 1).

Considerando a finalidade, as estratégias identificadas para garantir o acesso físico ou financeiro aos alimentos foram: Renda Mínima, PNAE, Restaurantes Populares, cozinhas comunitárias, distribuição/doação de cestas básicas e PAA.
Algumas estratégias apresentaram particularidades que mereceram destaque, conforme Quadro 2.

\section{Programas de transferência de renda ou renda básica emergencial}

Os Programas de transferência de renda ou renda mínima são alternativas para o combate à fome e a pobreza, e buscam assegurar a manutenção das necessidades básicas diárias. Com a pandemia, milhões de brasileiros tiveram seus vencimentos reduzidos ou perderam suas fontes de renda. Para assegurar a manutenção das necessidades básicas da população, o governo federal implementou um auxílio emergencial, chamado de renda básica emergencial, para desemprega-

Quadro 1. Ações/estratégias governamentais para garantia de SAN frente à Covid-19 no âmbito dos estados e capitais brasileiras, 2020.

\begin{tabular}{|c|c|c|c|}
\hline Ações/Estratégias & Formato & Estados & Capitais \\
\hline $\begin{array}{l}\text { Transferência de } \\
\text { renda }\end{array}$ & Auxílio emergencial & $\mathrm{AM}, \mathrm{AP}, \mathrm{BA}, \mathrm{DF}, \mathrm{RO}, \mathrm{SE}$ & -- \\
\hline \multirow[t]{5}{*}{$\begin{array}{l}\text { Programa de } \\
\text { Aquisição de } \\
\text { Alimentos (PAA) }\end{array}$} & $\begin{array}{l}\text { Compra com doação } \\
\text { simultânea }\end{array}$ & $\begin{array}{l}\text { AL, AP, BA, CE, DF, ES, GO, } \\
\text { MA, MT, PB, PE, PR, RJ, RS, } \\
\text { SC, SP, RN, MG }\end{array}$ & -- \\
\hline & Compra Institucional & GO, PE, SC & -- \\
\hline & PAA leite & $\begin{array}{l}\text { RO, MG, PB, CE, PE, BA, MA, } \\
\text { PI, AL, SE, RN, PR }\end{array}$ & -- \\
\hline & Formação de estoques & BA, RJ, SP & -- \\
\hline & Aquisição de sementes & $\mathrm{AL}, \mathrm{AM}$ & -- \\
\hline \multirow[t]{3}{*}{$\begin{array}{l}\text { Programa } \\
\text { Nacional de } \\
\text { Alimentação } \\
\text { Escolar (PNAE) }\end{array}$} & Kits de Alimentos & $\begin{array}{l}\text { AC, RR, } \mathrm{AL}^{2}, \mathrm{MT}, \mathrm{ES}, \mathrm{PR}, \mathrm{SC}, \\
\mathrm{RS}, \mathrm{AM}, \mathrm{TO}, \mathrm{AP}, \mathrm{RN}, \mathrm{PB}, \mathrm{MS}, \\
\mathrm{RO}\end{array}$ & $\begin{array}{l}\text { AC, AL, AP, BA, CE, ES, GO, } \\
\text { MA, MT, MS, MG, PA, PR }{ }^{1}, \mathrm{PE} \text {, } \\
\text { PI, RJ' }^{1} \text {, RN, RO, RS' }, \text { RR, SE, } \\
\text { TO, AM, SC }\end{array}$ \\
\hline & Auxílio financeiro & $\begin{array}{l}\text { BA, CE, GO, MG, PA, PE, PI, } \\
\text { SP, DF, RO }\end{array}$ & $\mathrm{AM}, \mathrm{SC}, \mathrm{SP}$ \\
\hline & $\begin{array}{l}\text { Manutenção das } \\
\text { Refeições nas escolas }\end{array}$ & -- & $\mathrm{PB}$ \\
\hline \multirow[t]{3}{*}{$\begin{array}{l}\text { Distribuição de } \\
\text { alimentos }\end{array}$} & Cestas básicas & $\begin{array}{l}\text { AL, AM, BA, CE, ES, GO, MA, } \\
\text { MT, MS, MG, PA, PB }{ }^{3}, P E, P I, \\
\text { RJ, RN, RS, RO, RR, SP, SE } \\
\text { TO }\end{array}$ & $\begin{array}{l}\text { AC, AL, AM, AP, BA, CE, ES, } \\
\text { GO, MA, MT, MS, MG }{ }^{3}, P A \\
\text { PB, PE, PI, PR, RJ, RN, RS, RO, } \\
\text { RR, SC }{ }^{3}, \text { SP, SE, TO }\end{array}$ \\
\hline & $\begin{array}{l}\text { Cartão/Voucher } \\
\text { Alimentação }\end{array}$ & $\mathrm{AP}, \mathrm{DF}, \mathrm{PB}^{3}, \mathrm{PR}, \mathrm{SE}^{3}$ & $\mathrm{SC}^{3}, \mathrm{MG}^{3}$ \\
\hline & $\begin{array}{l}\text { Equipamentos públicos } \\
\text { de SAN (restaurantes } \\
\text { populares, cozinhas } \\
\text { comunitárias, banco de } \\
\text { alimentos) }\end{array}$ & $\begin{array}{l}\mathrm{AL}, \mathrm{AM}^{5}, \mathrm{BA}, \mathrm{MA}, \mathrm{PA}, \mathrm{PB}, \mathrm{PI}^{4}, \\
\mathrm{RJ}, \mathrm{RN}, \mathrm{RS}^{4}, \mathrm{SE}, \mathrm{SP}\end{array}$ & $\begin{array}{l}\mathrm{AL}, \mathrm{AP}, \mathrm{AM}^{5}, \mathrm{BA}, \mathrm{CE}, \mathrm{MT}, \mathrm{MG} \\
\mathrm{PA}, \mathrm{PB}^{5}, \mathrm{PE}, \mathrm{RJ}, \mathrm{SP}^{5}, \mathrm{TO}\end{array}$ \\
\hline
\end{tabular}

${ }^{1}$ Considerou-se apenas a estratégia que estava em vigor no período da coleta de dados. ${ }^{2}$ Apenas distribuição de alimentos que estavam estocados em algumas escolas no início da suspensão das atividades. ${ }^{3}$ Associação de distribuição de cestas básicas com cartão alimentação. ${ }^{4}$ Únicos locais que citaram bancos de alimentos. ${ }^{5}$ Unicos locais que citaram cozinhas comunitárias. 
Quadro 2. Singularidades identificadas nas estratégias governamentais para garantia do DHAA frente à pandemia de Covid-19, 2020.

\begin{tabular}{|l|l|l|}
\hline \multicolumn{1}{|c|}{ Singularidade } & \multicolumn{1}{|c|}{ Estados } & \multicolumn{1}{c|}{ Capitais } \\
\hline $\begin{array}{l}\text { Focalização da assistência aos } \\
\text { estudantes }\end{array}$ & $\begin{array}{l}\text { AC, AL, ES, RR, MT, PR, RS, SC, } \\
\text { DF, GO, MG, PE, PI, SP, RO }\end{array}$ & $\begin{array}{l}\text { AC, AP, ES, MT, MS, MG }{ }^{2}, \text { PA, PR, } \\
\text { PI, RJ, RS }{ }^{3}, \text { RR, SC, TO, AM, SC, SP }\end{array}$ \\
\hline $\begin{array}{l}\text { Parcerias Público-Privadas na doação } \\
\text { de alimentos }\end{array}$ & $\begin{array}{l}\text { AM, ES, MG, MS, PA, PI, RJ, RO, } \\
\text { RN, RS, SE, SP }\end{array}$ & $\begin{array}{l}\text { AC, AP, AM, MS, PA, PB, PR, RJ, } \\
\text { RS, SP }\end{array}$ \\
\hline $\begin{array}{l}\text { Uso de recursos do Fundo de Combate } \\
\text { à Pobreza para ações de SAN }\end{array}$ & $\begin{array}{l}\text { AL, CE, PB, PE, PR, RN, SE, SP, } \\
\text { TO }\end{array}$ & -- \\
\hline Criação de PAA local & $\begin{array}{l}\text { AL, AM, AP, BA, CE, DF, ES, GO, } \\
\text { MA, MG, MT, PB, PE, PI, PR, RJ, } \\
\text { RN, RO, RS, SC, SP }\end{array}$ & \\
\hline
\end{tabular}

${ }^{1}$ Rondônia: Kits de alimentos para estudantes das escolas indígenas e auxílio financeiro para estudantes em vulnerabilidade social. ${ }^{2}$ Belo Horizonte: Entrega de apenas um kit de alimentos por família, independentemente do número de estudantes que a família possuir e da mesma estar em situação de vulnerabilidade social. ${ }^{3}$ Porto Alegre: Entrega de kits de alimentos apenas para estudantes da educação infantil. *As capitais geralmente são beneficiadas com o PAA da agricultura familiar promovido pelos seus Estados.

dos, autônomos e trabalhadores formais e informais que ficaram impossibilitados de desenvolver suas atividades e não são beneficiários de outros programas de transferência de renda ${ }^{9,10}$.

Inicialmente, o valor proposto pela Presidência da República foi de $\mathrm{R} \$ 200$, passando para $\mathrm{R} \$$ 600,00 após contestações de lideranças políticas. O recurso foi assegurado inicialmente por três meses ${ }^{9}$, sendo prorrogado por mais dois devido à demora no retorno das atividades econômicas, estando em debate sua continuidade após o pagamento da quinta parcela ${ }^{11}$.

Outra medida adotada para atender as necessidades da população pobre e extremamente pobre durante a pandemia foi a adequação dos valores repassados aos titulares do PBF e a alteração dos critérios para recebimento do BPC, principais programas de transferência de renda atualmente implementados no país.

O BPC contempla idosos acima de 65 anos ou portadores de deficiência, com renda mínima inferior a $1 / 4$ de salário mínimo e não vinculados a nenhum outro benefício ou regime da previdência social. Com a pandemia, foi ampliada de $25 \%$ para 50\% de um salário mínimo per capita a renda mínima necessária para recebimento do benefício, provendo um maior número de famílias ${ }^{12}$.

$O$ PBF beneficia mensalmente famílias extremamente pobres (renda mensal de até R\$ $89,00 /$ pessoa) ou pobres (renda mensal de até R\$ 178,00/pessoa), com R\$ 89,00 fixo, vinculado ao cumprimento de condicionalidades que fomentam o acesso à saúde e à educação. Um benefício variável (R\$ 41,00 ou 48,00) é atribuído a famílias com gestantes, crianças ou adolescentes até 17 anos, podendo cada família acumular até 5 be- nefícios mensalmente ${ }^{13}$. Com a pandemia, o valor equiparou-se temporariamente aos $\mathrm{R} \$ 600,00$ disponibilizados pelo auxílio emergencial, sendo de $\mathrm{R} \$ 1.200,00$ para mulheres que sustentam sozinhas a família9.

O DF e alguns estados do Norte e Nordeste disponibilizaram auxílio emergencial próprio para indivíduos ou famílias de baixa renda não cadastrados em programas de transferência de renda do governo federal. Os valores variam de $\mathrm{R} \$ 100,00$ a 408,00 mensais, durando de três meses a enquanto permanecer a pandemia ${ }^{14}$.

\section{Programa Nacional de Alimentação Escolar (PNAE)}

O PNAE é destinado à oferta de alimentação adequada e saudável e ações de educação alimentar e nutricional a estudantes da educação básica pública. Por meio do programa, o governo federal repassa a estados, municípios e escolas federais recursos para compor o orçamento para compras de gêneros alimentícios durante o ano letivo, conforme número de matriculados. O PNAE considera que a alimentação escolar é direito de todos os alunos da educação básica pública e dever do Estado, e objetiva garantir a SAN, com acesso igualitário, priorizando os mais vulnerabilizados ${ }^{15}$.

O fechamento das escolas a partir de março/2020 devido à pandemia deixou um imenso contingente de estudantes sem acesso às refeições diárias fornecidas pelo PNAE, contribuindo para a InSAN de muitos dos escolares e suas famílias. Como consequência, o marco legal do programa foi alterado, autorizando que, durante a suspen- 
são das aulas devido a emergência/calamidade pública, a distribuição de alimentos adquiridos com recursos do PNAE possa ser feita diretamente aos pais ou responsáveis dos estudantes, a critério do poder público local ${ }^{16}$.

A execução do PNAE durante esse período tem se dado mediante a entrega de kits de alimentos e pagamento de auxílio financeiro. Também foi observada a manutenção do fornecimento de refeições nas escolas.

No total, 23 estados, 25 capitais e o DF desenvolveram alguma ação no primeiro semestre de 2020 relacionada ao PNAE no contexto da pandemia. Destes, 21 capitais e 14 estados adotaram a distribuição de kits de alimentos, enquanto 8 estados, o DF e o município de São Paulo adotaram o pagamento de auxílio financeiro. Três capitais (Manaus, Porto Velho e Florianópolis) adotaram simultaneamente ambas as estratégias. João Pessoa adotou estratégia própria, retomando em junho o fornecimento de refeições nas demais escolas da rede municipal ${ }^{17}$ (Quadro 1). Não foram identificadas medidas de assistência aos estudantes nos estados do Maranhão, Rio de Janeiro e Sergipe.

O auxílio variou entre $\mathrm{R} \$ 50,00$ e 179,10, sendo concedido mediante transferência bancária diretamente aos responsáveis ou entregue via vale-compras (cartão magnético) para aquisição de alimentos em estabelecimentos credenciados. Os governos devem utilizar recursos próprios para pagamento do auxílio, uma vez que o FNDE é exclusivo para compra de gêneros alimentícios, o que pode explicar a variação nos valores ${ }^{18}$. As informações disponíveis nos sites oficiais não explicitaram a origem dos recursos utilizados.

Foi predominante nos dois formatos a cobertura focalizada, direcionada apenas a estudantes de famílias em situação de extrema pobreza, comprovada por meio do registro no CadÚnico ou participação no PBF.

Quanto ao início de execução das estratégias, apenas 22 estados e capitais iniciaram ações de assistência alimentar aos estudantes entre março e abril.

Não foi possível analisar o cumprimento efetivo das medidas divulgadas a partir das fontes consultadas.

\section{Distribuição de alimentos}

Quanto à distribuição de alimentos, foram identificadas estratégias de distribuição de refeições subsidiadas por meio dos restaurantes populares (RP) e cozinhas comunitárias, assim como o fornecimento de cestas básicas, com entrega direta dos alimentos ou cartão com valor específico, exclusivo para compra de alimentos.

Os RP e cozinhas comunitárias fazem parte da Rede de Equipamentos Públicos de SAN, que buscam ampliar a oferta de refeições nutricionalmente adequadas, com preços acessíveis à população de baixa renda, vulnerabilizada socialmente e em situação de InSAN. Embora de acesso universal, o público de cobertura desses equipamentos são pessoas em situação de rua, trabalhadores informais e desempregados. Para os mais vulnerabilizados, as refeições podem ser distribuídas gratuitamente ou por um valor simbólico, e para os demais usuários o valor da refeição é estabelecido localmente ${ }^{19}$. Devido à ausência de financiamento específico, apenas 12 estados e 13 capitais apresentaram algum equipamento de SAN desenvolvendo assistência especial durante a pandemia (Quadro 1). Capitais como Curitiba e João Pessoa abriram equipamentos novos para ampliar assistência ao público vulnerabilizado. A prefeitura de São Paulo criou um programa em parceria com entidades da sociedade civil e de apoio à população vulnerabilizada, focando geração de renda e movimentação da economia local $^{20}$.

A distribuição de cestas básicas foi priorizada por todos os governos estaduais e municipais como medida emergencial, mediante a entrega direta dos alimentos ou cartão-alimentação (Quadro 1). A prefeitura de Belo Horizonte se destacou com um sistema organizado de entrega de cestas, com abrangência estimada em 1 milhão de pessoas e parceria firmada com 160 supermercados $^{21}$. Florianópolis também estabeleceu parcerias com estabelecimentos para disponibilizar alimentos via cartão-alimentação com pagamento via aplicativo ou liberação da compra por telefonema ${ }^{22}$.

A maioria das gestões estabeleceu parcerias público-privadas (PPP) para fins de arrecadação de recursos financeiros ou gêneros alimentícios junto a empresas diversas e a sociedade civil (Quadro 1). No Acre foi realizada uma live onde o governador atendeu aos telefonemas dos doadores, arrecadando mais de 90 toneladas de alimentos.

Alguns estados têm utilizado o Fundo de Combate à Pobreza (FCP) como fonte de recursos para compra dos alimentos (Quadro 2). As arrecadações do fundo direcionam recursos públicos para programas sociais voltados à nutrição, habitação, educação e saúde. No Rio Grande do Norte $^{23}$, os recursos do FCP são usados para 
manter os RP. No Paraná, o recurso foi usado na compra de alimentos da agricultura familiar para composição de cestas básicas, e para pagamento de auxílio de $\mathrm{R} \$ 50,00$ por três meses para famílias vulnerabilizadas comprar alimentos em estabelecimentos credenciados ${ }^{24}$.

São Paulo, estado mais populoso do país, instituiu programa para distribuir 1 milhão de cestas de alimentos a pessoas em situação de extrema vulnerabilidade social. Segundo o governo, o investimento de mais de $\mathrm{R} \$ 100$ milhões vem do setor privado ${ }^{25}$. Contudo, ressaltam o uso do FCP para aquisição de alimentos da agricultura familiar e confecção de 10 mil cestas básicas destinadas às famílias carentes ${ }^{26}$, com priorização de investimentos nos agricultores familiares assentados e remanescentes de quilombos.

\section{Programa de Aquisição de Alimentos (PAA)}

O PAA promove o acesso à alimentação e incentiva a agricultura familiar por meio da compra de alimentos da agricultura camponesa pelo Estado, contribuindo para a constituição de estoques públicos de alimentos. O programa é executado por estados e municípios, em parceria com o Ministério da Cidadania e pela Companhia Nacional de Abastecimento (Conab), por meio de cinco modalidades: compra com doação simultânea, compra institucional, PAA leite, formação de estoques e aquisição de sementes ${ }^{27}$.

Iniciativas de governos estaduais para aquisição de produtos da agricultura familiar estão em andamento devido à pandemia. Alguns estados potencializaram os programas existentes, outros estabeleceram ações emergenciais para aquisição de alimentos da agricultura familiar para posterior doação a pessoas em InSAN. Nesse cenário, uma das medidas executadas pela Conab foi a prorrogação dos projetos do PAA que venceram a partir de dezembro de 2019, mas ainda possuíam saldo a vencer até junho de 2020. Com isso, agricultores beneficiados ganharam mais tempo para entregar alimentos às organizações pelo país ${ }^{28}$.

Em abril de 2020 a Conab anunciou uma suplementação orçamentária para o PAA, com previsão de aporte de $\mathrm{R} \$ 220$ milhões para compra com doação simultânea. A Conab ainda conta com o orçamento de aproximadamente $\mathrm{R} \$ 10$ milhões, provenientes de 21 emendas parlamentares, a ser destinado a projetos no Amapá, Amazonas, Goiás, Maranhão, Minas Gerais, Pernambuco, Piauí, Rio de Janeiro, Roraima, Sergipe, Tocantins e DF, devendo atender cerca de 1.500 agricultores familiares nesses estados ${ }^{28}$.
A compra com doação simultânea de alimentos da agricultura familiar para famílias em situação de InSAN foi registrada até junho de $2020 \mathrm{em}$ ao menos 15 estados, mas em geral relacionadas a contratos assinados antes da pandemia, em 2019 (Quadro 1). A compra institucional também foi registrada em Goiás, mas sem caracterizar ação específica para o enfrentamento dos impactos da Covid-1928.

O apoio à formação de estoques, embora não tenha sido estruturado como uma ação específica para o enfrentamento da pandemia, foi registrado em 2020 em cinco estados (Quadro 1), onde pequenos agricultores receberam recursos para comercializar seus produtos, referentes a contratos assinados em $2019^{28}$.

A distribuição de sementes para famílias de pequenos produtores foi registrada em Alagoas e no Amazonas ${ }^{28}$, também referente a chamadas públicas de 2019. Em julho, o Governo Federal abriu crédito de $\mathrm{R} \$ 156$ milhões para a compra de leite para enfrentamento da pandemia.

Considerando especificamente comunidades tradicionais, no estado do Amazonas, a Nota Técnica Conjunta ADAF/SFA-AM/5ㅇ Ofício-PR -AM possibilitou compra direta de proteínas e processados vegetais de povos indígenas com produção excedente, gerando renda e reduzindo a InSAN na região ${ }^{29}$.

Os estados do Maranhão, Espírito Santo, Minas Gerais e Rio Grande do Norte não lançaram planos específicos de enfrentamento à pandemia envolvendo o $\mathrm{PAA}^{30}$.

\section{Discussão}

A pandemia de Covid-19 impôs desafios sem precedentes para os sistemas alimentares em todo o mundo. A crise econômica e social associada à emergência sanitária exacerba as iniquidades sociais e ameaça a SAN, impulsionando o crescimento da pobreza e desvelando a iminência da crise da fome $e^{31,32}$.

Os impactos da pandemia para quem já vive em situação de InSAN, como a população em situação de rua, aqueles abaixo da linha da pobreza, boa parte da população rural e povos e comunidades tradicionais, podem ser "catastróficos", com redução de acesso aos alimentos e à renda necessária para aquisição de outros itens de primeira necessidade, aumento das tensões sociais e conflitos, migração, violência, desnutrição severa e morte ${ }^{31}$.

Entidades e organismos internacionais apontam a necessidade de desenvolver ações em três 
áreas centrais: a) manter o fornecimento de alimentos para a população, incluindo adotar medidas para facilitar o escoamento de alimentos produzidos pelos pequenos produtores; b) apoiar os mais vulneráveis, assegurando acesso a formas de produção e a alimentos adequados e saudáveis; c) investir em sistemas alimentares sustentáveis e resilientes ${ }^{31,33}$.

No Brasil, a crise sanitária emerge em um cenário de piora dos indicadores sociais e desmonte de políticas públicas, como as de proteção social e trabalhistas. Vários fatores agravam os impactos da pandemia no país, e estão ligados a causas estruturais de fome e desnutrição, como grilagem de terras, uso de agrotóxicos, promoção de alimentos ultraprocessados e industrializados, privatização e financeirização do setor saúde e aprofundamento das desigualdades ${ }^{34}$. O país vive um momento de estagnação econômica e paralisação dos programas de SAN, o que aumenta a população vulnerabilizada, exigindo do governo a adoção de medidas para proteção desses grupos.

Embora importantes, foram registradas limitações nas iniciativas adotadas, bem como nos dados disponíveis nos sites dos governos estaduais e municipais. Em geral, as informações são disponibilizadas como notícias, de forma dispersa, frequentemente não citando o dispositivo legal que as institui e regula.

\section{Programas de transferência de renda ou renda mínima}

As consequências da pandemia sobre os sistemas alimentares afetam de modo particular os mais pobres, que gastam parcela significativa de seus rendimentos com comida, situação que tende a se agravar com o aumento do subemprego e do desemprego decorrentes da pandemia, que afeta o fornecimento e aquisição de alimentos ${ }^{35}$.

Isso reforça a importância de estruturar políticas públicas específicas para assegurar que alimentos de qualidade cheguem à população, bem como para que os produtores, especialmente os pequenos, tenham acesso à uma renda mínima durante a pandemia, investindo na estruturação e fortalecimento de sistemas de proteção social abrangentes e universais ${ }^{32,35}$.

No Brasil, embora a principal iniciativa adotada pelo governo federal tenha sido o repasse financeiro para os mais pobres, assim como em outros países afetados pela pandemia ${ }^{35}$, dificuldades para acessar o auxílio emergencial foram observadas. Uma refere-se à exigência da realização de um cadastro on-line para obtenção do be- nefício, embora 26\% dos brasileiros continuem desconectados e $16 \%$ dos analfabetos ou com baixa escolaridade não usem a internet ${ }^{36}$. Igualmente, 46 milhões de brasileiros vivem sem conta bancária, acesso à internet e Cadastro de Pessoa Física (CPF) ativo, não sendo possível acessar aplicativos ou sacar o dinheiro em bancos.

Ainda, o presidente vetou o pagamento do auxílio a grupos vulnerabilizados, como pescadores artesanais, agricultores familiares, assentados da reforma agrária, taxistas, motoristas e entregadores de aplicativo, entre outros ${ }^{9}$. Em agosto, foi vetado o pagamento de benefício a agricultores familiares durante a pandemia, aumentando o risco da fome nesse grupo ${ }^{37}$.

Outro problema refere-se à demora no pagamento do benefício aos que conseguiram cumprir todas as exigências cadastrais. Pesquisa com moradores de favelas de todos os estados brasileiros revela que, até junho, 41\% dos que solicitaram o auxílio emergencial ainda não receberam nenhuma parcela, e que $80 \%$ das famílias estão sobrevivendo com menos da metade da renda que tinham antes da pandemia ${ }^{38}$.

É necessário adequar as exigências para obtenção do auxílio, conforme particularidades e limitações desses grupos. Por exemplo, povos e comunidades tradicionais, que já vivem em condições de grave InSAN, encontram dificuldades no acesso à tecnologia e de deslocamento para receber os valores a que têm direito. Logo, é importante estruturar alternativas como a ampliação dos prazos para saque dos benefícios e a facilitação do acesso em áreas remotas, garantindo seus direitos sociais e previdenciários ${ }^{29}$.

O Ministério Público Federal (MPF) ajuizou uma ação civil pública para que sejam adotadas medidas emergenciais em favor de povos indígenas, quilombolas e comunidades tradicionais no Amazonas para facilitar o acesso a benefícios sociais e previdenciários concedidos pelo governo federal, com o objetivo de garantir SAN a esses grupos enquanto vigoram as medidas de isolamento social devido à pandemia ${ }^{29}$.

Reitera-se a essencialidade da concessão do auxílio emergencial e dos programas sociais continuados de transferência de renda, uma vez que esse dinheiro movimenta a economia e assegura a aquisição de alimentos.

\section{Programa Nacional de Alimentação Escolar}

Devido ao fechamento das escolas em pelo menos 197 países, mais de 369 milhões de crianças não estão recebendo alimentação escolar no 
mundo, sendo 40,1 milhões destas brasileiras ${ }^{39}$. Em algumas regiões, o fechamento de escolas reduziu o acesso a alimentos para crianças ou substituiu os programas de alimentação escolar por provisão inadequada de alimentos ${ }^{34}$.

A aquisição de alimentos de pequenos produtores e ou associações para assegurar a continuidade da distribuição de refeições aos estudantes durante o período do fechamento das escolas é uma das principais medidas recomendadas para mitigar os impactos da pandemia ${ }^{33}$. O cumprimento dos parâmetros nutricionais estabelecidos no PNAE e a manutenção das compras públicas de alimentos da agricultura familiar são fundamentais para o fornecimento de uma alimentação adequada e saudável aos escolares ${ }^{40}$ e para o escoamento da produção e a geração de renda para os agricultores.

No Brasil, apesar do repasse do auxílio financeiro às famílias facilitar a logística para assistência aos estudantes, a medida não assegura o acesso aos melhores alimentos em termos de quantidade e qualidade. O auxílio financeiro, em vários casos limitado a alguns estabelecimentos credenciados, não se compara às possibilidades das grandes compras públicas realizadas em atacado ${ }^{41}$.

A focalização em detrimento da assistência universal aos estudantes em diversos estados também é preocupante. $\mathrm{O}$ agravamento da crise econômica pela pandemia levou muitas famílias à situação de pobreza e pobreza extrema. Estima-se que grande parte destas sequer estão cadastradas no CadÚnico, o que as exclui de acessar algumas medidas de assistência emergencial, como a alimentação escolar em alguns estados e municípios $^{42}$.

Essas limitações impedem o acesso amplo ao programa, bem como a alimentos de qualidade. Ainda, em alguns estados e capitais não foram observadas quaisquer medidas vinculadas ao PNAE, levantando preocupações sobre a SAN.

\section{Distribuição de Alimentos}

A distribuição de alimentos por meio de programas sociais, particularmente em países Latino -Americanos, tem assegurado comida a milhões de famílias de baixa renda, principalmente via distribuição de refeições e renda para aquisição de alimentos ${ }^{43}$.

Dentre estas estratégias, os RP destacam-se como via assistencial de alguns estados e municípios à população em situação de rua e trabalhadores de baixa renda. Com a pandemia, os RP se tornaram equipamentos de SAN ainda mais estratégicos para o público vulnerabilizado, demonstrando a importância de ampliar a iniciativa e investir em seu financiamento continuado. $\mathrm{O}$ país conta atualmente com apenas $135 \mathrm{RP}$, capazes de servir até mil refeições/dia ${ }^{19}$, número insuficiente para atender à demanda. A identificação de poucas cozinhas comunitárias e bancos de alimentos expõe a subutilização dos equipamentos de SAN em estados/municípios.

Quanto às cestas básicas, observa-se a padronização dos itens alimentícios, descaracterizando a cultura alimentar local, principalmente singularidades de povos tradicionais. Os produtos dessas cestas vêm sendo adquiridos por meio de Termo firmado com o Ministério da Mulher, da Família e dos Direitos Humanos (MMFDH), a partir da abertura de crédito extraordinário em favor da Presidência e alguns ministérios. Dos R\$ 639.034.512,00 liberados, apenas 7,04\% foram destinados ao MMFDH, cujas ações de enfrentamento à pandemia envolvem diversas iniciativas, sendo a única diretamente voltada à SAN o "abastecimento com gêneros alimentícios e higiene dos povos e comunidades tradicionais" ${ }^{44}$.

Para estes povos, a estimativa é de 323,4 mil cestas durante a pandemia, sem previsão de outras compras no resto do ano. Embora investimentos na assistência a povos tradicionais venham diminuindo anualmente, com a pandemia, a União, junto com a Conab, tem realizado a distribuição de cestas ${ }^{29}$.

O fornecimento de refeições e alimentos é recomendado internacionalmente como estratégia para manter o acesso à comida no contexto da pandemia, apontando que os governos devem fortalecer os mecanismos de proteção social e os programas de assistência alimentar emergenciais. Contudo, a distribuição e subsídios para alimentos e refeições prontas segue como uma estratégia pontual para assistência a grupos específicos, caracterizando ações direcionadas ao consumo sem interferir na cadeia produtiva. Esse tipo de iniciativa até pode reduzir os impactos das iniquidades sociais, mas atua superficialmente nas causas das desigualdades ${ }^{45}$.

Muitas das ações implementadas foram estabelecidas mediante parcerias público-privadas (PPP), com doações da sociedade civil ancorados no terceiro setor, em processos por vezes permeados por conflitos de interesse e desrespeito à legislação trabalhista ${ }^{46}$.

Ainda sobre a malversação do uso dos recursos, em Pernambuco, a utilização do FCP para compras das cestas básicas é investigada por suspeita de contratação de empresa fantasma ${ }^{47}$. Já 
em Alagoas, o uso do FCP só se deu sob pressão política da Assembleia Legislativa e de organizações da sociedade civil junto ao governo ${ }^{48}$.

\section{Programa de Aquisição de Alimentos}

O abastecimento alimentar por meio de compras governamentais de alimentos é uma importante estratégia de promoção da SAN, que fortalece circuitos locais e regionais e redes de comercialização; valoriza a biodiversidade e a produção orgânica e agroecológica de alimentos; incentiva hábitos alimentares saudáveis e estimula o cooperativismo e o associativismo. Sua vinculação a iniciativas como o PNAE minimiza a crise gerada pela pandemia, garantindo alimentos in natura à população vulnerabilizada. Essas estratégias asseguram a distribuição da produção da agricultura familiar, dado que o acesso aos mercados foi profundamente afetado pela pandemia. Medidas similares foram recomendadas internacionalmente, e envolvem logística para estabilizar a oferta de produtos da agricultura familiar, assegurando transporte e comercialização dos alimentos e minimizando a exposição ao vírus ${ }^{33,43}$.

Embora o PAA tenha um grande potencial para reduzir a InSAN, o programa é insuficientemente utilizado. A aquisição de sementes, por exemplo, foi operacionalizada em alguns estados, mas não enquanto estratégia para o enfrentamento da pandemia. Para assegurar a SAN no contexto da Covid-19, organismos internacionais recomendam que a modalidade seja uma ação prioritária ${ }^{31}$.

A compra com doação simultânea de alimentos gera renda ao produtor familiar e atende a entidades e organizações para promoção da SAN. Essa iniciativa não é voltada especificamente para o enfrentamento dos impactos da Covid-19, pois refere-se em geral a contratos anteriores à pandemia.

O PAA é uma das principais políticas de apoio e estímulo à agricultura familiar no Brasil, e a ampliação de incentivos governamentais para seu fortalecimento deveria ser prioritária. Frente à necessidade de fortalecer compras públicas para inclusão social e produtiva rural, o Ministério da Cidadania propôs a distribuição de orçamento adicional para enfrentamento da crise gerada pela pandemia entre as modalidades PAA-Leite e compra com doação simultânea ${ }^{49}$.
Diferentemente das iniciativas de doação direta de alimentos, o PAA gera renda, respeita a cultura alimentar, promove soberania, estimula o consumo de alimentos de qualidade, fortalece circuitos curtos de produção e consumo e promove desenvolvimento territorial. Essas características tornam o programa com maior potencial de promover o "acesso regular e permanente a alimentos de qualidade, em quantidade suficiente, sem comprometer o acesso a outras necessidades essenciais, tendo como base práticas alimentares promotoras de saúde que respeitem a diversidade cultural e que sejam ambiental, cultural, econômica e socialmente sustentáveis", direitos previstos no Sistema Nacional de Segurança Alimentar e Nutricional ${ }^{50}$.

\section{Considerações finais}

Evidenciou-se que as medidas governamentais adotadas no Brasil não são suficientes para evitar a InSAN decorrente da pandemia. Considerando as iniciativas que contribuem para a SAN, observou-se no país uma prevalência de ações direcionadas ao consumo, sem interferir na cadeia produtiva. Também foi evidenciada a necessidade de prestar assistência específica a grupos em maior situação de vulnerabilidade, compatíveis com as diferenças socioterritoriais, assegurando que necessidades nutricionais específicas sejam plenamente atendidas.

A crise sanitária revela a violação de direitos humanos fundamentais, como o direito humano à alimentação adequada e saudável, expondo as profundas injustiças dos sistemas alimentares, assim como a necessidade de estruturar políticas públicas que assegurem, além do fornecimento de alimentos, condições suficientes para manutenção da dignidade humana, a despeito das questões de gênero, raça/etnicidade e classe.

A pandemia evidencia a necessidade urgente da adoção de medidas coordenadas nas escalas global, nacional e local para prevenir a crise humanitária e alimentar iminente, que ameaça particularmente grupos em maior situação de vulnerabilidade. Deve-se implementar e fortalecer as iniciativas voltadas à proteção social, assegurando o pleno acesso à alimentação saudável e adequada. 


\section{Colaboradores}

AM Gurgel trabalhou na concepção e design do trabalho; forneceu contribuições substanciais para a coleta, análise e interpretação de dados; atuou na elaboração do manuscrito e revisão crítica do conteúdo intelectual; e na revisão e aprovação final da versão submetida. CCS Santos forneceu contribuições substanciais para a coleta, análise e interpretação de dados; atuou na elaboração do manuscrito e revisão crítica do conteúdo intelectual; e na revisão e aprovação final da versão submetida. KPS Alves, VS Leal e JM Araujo forneceram contribuições substanciais para a coleta, análise e interpretação de dados; atuaram na elaboração do manuscrito; e na revisão e aprovação final da versão submetida.

\section{Referências}

1. Organização Pan-Americana de Saúde (OPAS). Folha informativa - COVID-19 [Internet]. Brasília: OPAS; 2020 [acessado 2020 Maio 13]. Disponível em: https:// tinyurl.com/rz9mdv6

2. Centro Europeu de Prevenção e Controle de Doenças (ECDC). COVID-19 situation update worldwide [Internet]. Solna: ECDC; 2020 [acessado 2020 Maio 13]. Disponível em: https://tinyurl.com/w9y39vp

3. Wang Z, Tang K. Combating COVID-19: health equity matters. Nature 2020; 26:458.

4. Wenham C, Smith J, Morgan R. COVID-19: the gendered impacts of the outbreak. Lancet 2020; 395:846848.

5. Oliveira TC, Abranches MV, Lana RM. (In)Segurança alimentar no contexto da pandemia por SARS-CoV-2. Cad Saude Publica 2020; 36(4):e00055220.

6. Reis-Filho JA, Quinto D. COVID-19, Afastamento social, pesca artesanal e segurança alimentar: como esses temas estão relacionados e quão importante é a soberania dos trabalhadores da pesca diante do cenário distópico. SciELO 2020 [preprint].

7. Recine E, Pacheco ME. A urgência do combate à fome [Internet]. Folha de São Paulo; 2020 [acessado 2020 Maio 15]. Disponível em: https://tinyurl.com/ y6gy52b7

8. Bardin L. Análise de conteúdo. Lisboa: Edições 70; 1995.

9. Brasil. Lei $\mathrm{n}^{\circ} 13.982$, de 2 de abril de 2020 . Altera a Lei $n^{\circ} 8.742$, de 7 de dezembro de 1993, para dispor sobre parâmetros adicionais de caracterização da situação de vulnerabilidade social para fins de elegibilidade ao benefício de prestação continuada (BPC), e estabelece medidas excepcionais de proteção social a serem adotadas durante o período de enfrentamento da emergência de saúde pública de importância internacional decorrente do coronavírus (Covid-19) responsável pelo surto de 2019, a que se refere a Lei no 13.979 , de 6 de fevereiro de 2020. Diário Oficial da União; 2020.

10. Brasil. Lei $n^{\circ} 13.998$, de 14 de maio de 2020. Promove mudanças no auxílio emergencial instituído pela Lei no 13.982 , de 2 de abril de 2020; e dá outras providências. Diário Oficial da União; 2020.

11. Brasil. Decreto $n^{\circ} 10.412$, de 30 de junho de 2020. Altera o Decreto no 10.316 , de 7 de abril de 2020, para prorrogar o período de pagamento do auxílio emergencial de que trata a Lei no 13.982 , de 2 de abril de 2020. Diário Oficial da União; 2020.

12. Brasil. Lei $n^{\circ} 13.981$, de 23 de março de 2020. Altera a Lei no 8.742, de 7 de dezembro de 1993 (Lei Orgânica da Assistência Social), para elevar o limite de renda familiar per capita para fins de concessão do benefício de prestação continuada. Diário Oficial da União; 2020.

13. Brasil. Decreto $\mathrm{n}^{\circ} 9.396$, de 30 de maio de 2018. Altera o Decreto no 5.209, de 17 de setembro de 2004, e o Decreto no 7.492, de 2 de junho de 2011, para reajustar valores referenciais de caracterização das situações de pobreza e de extrema pobreza e os de benefícios do Programa Bolsa Família. Diário Oficial da União; 2018.

14. Fundação Getúlio Vargas (FGV). O combate à Covid-19 no Brasil. Brasilia: FGV; 2020. 
15. Brasil. Lei $\mathrm{n}^{\circ} 11.947$, de 16 de junho de 2009. Dispõe sobre o atendimento da alimentação escolar e do Programa Dinheiro Direto na Escola aos alunos da educação básica; altera as Leis nos 10.880, de 9 de junho de 2004, 11.273, de 6 de fevereiro de 2006, 11.507, de 20 de julho de 2007; revoga dispositivos da Medida Provisória no 2.178-36, de 24 de agosto de 2001, e a Lei no 8.913, de 12 de julho de 1994; e dá outras providências. Diário Oficial da União; 2009.

16. Brasil. Lei $\mathrm{n}^{\circ} 13.987$, de 7 de abril de 2020. Altera a Lei $\mathrm{n}^{\circ} 11.947$, de 16 de junho de 2009, para autorizar, em caráter excepcional, durante o período de suspensão das aulas em razão de situação de emergência ou calamidade pública, a distribuição de gêneros alimentícios adquiridos com recursos do Programa Nacional de Alimentação Escolar (Pnae) aos pais ou responsáveis dos estudantes das escolas públicas de educação básica. Diário Oficial da União; 2020.

17. João Pessoa. Prefeitura de João Pessoa oferece refeiçães para 65 mil estudantes da rede municipal [Internet]. 2020 [acessado 2020 Ago 13]. Disponível em: https:// tinyurl.com/yy5ezy72

18. Brasil. Resolução $\mathrm{n}^{\circ}$ 2, de 9 de abril de 2020. Dispõe sobre a execução do Programa Nacional de Alimentação Escolar - PNAE durante o período de estado de calamidade pública, reconhecido pelo Decreto Legislativo $n^{\circ} 6$, de 20 de março de 2020 , e da emergência de saúde pública de importância internacional decorrente do novo coronavírus - Covid-19. Diário Oficial da União; 2020.

19. Brasil. Fazer refeição em Restaurante Popular [Internet]. 2020 [acessado 2020 Ago 13]. Disponível em: https://tinyurl.com/y6hmelfh

20. Cidade de São Paulo. Cozinhando pela Vida: programa inicia distribuição de 30 mil refeições para população em situação de vulnerabilidade [Internet]. 2020 [acessado 2020 Ago 13]. Disponível em: https://tinyurl. com/ycmfqk8p

21. Prefeitura de Belo Horizonte. Mais de 1 milhão de pessoas recebem cestas e kits de higiene oferecidos pela $P B H$ [Internet]. 2020 [acessado 2020 Ago 13]. Disponível em: https://tinyurl.com/yyq5bna4

22. Prefeitura de Florianópolis. Cartão-alimentação da Prefeitura de Florianópolis beneficiará 1223 autônomos sem renda [Internet]. 2020 [acessado 2020 Ago 13]. Disponível em: https://tinyurl.com/y3kn5qx3

23. Governo do Estado do Rio Grande do Norte. Restaurantes Populares deverão fornecer quentinhas e talheres descartáveis [Internet]. 2020 [acessado 2020 Ago 17]. Disponível em: https://tinyurl.com/y2xw6vbu

24. Paraná. Decreto $\mathrm{n}^{\circ} 4.570$, de 04 de maio de 2020. Regulamenta a Lei $\mathrm{n}^{\circ} 20.172$, de 7 de abril de 2020, que autorizou a concessão de auxílio emergencial, com recursos do FECOP, à pessoa economicamente vulnerabilizada em decorrência da emergência de saúde pública de importância internacional causada pela infecção humana pelo coronavírus (Covid-19). Diário Oficial do Estado; 2020.

25. Governo do Estado de São Paulo. Estado vai distribuir 1 milhão de cestas de alimentos para população em extrema pobreza [Internet]. 2020 [acessado 2020 Ago 13]. Disponível em: https://tinyurl.com/yymsr2r5
26. Governo do Estado de São Paulo. Governo de SP doa 10 mil cestas básicas para famílias carentes no Estado [Internet]. 2020 [acessado 2020 Ago 13]. Disponível em: https://tinyurl.com/yyagapd5

27. Brasil. PAA: 10 anos de aquisição de alimentos [Internet]. Brasília: Secretaria Nacional de Segurança Alimentar e Nutricional; 2020 [acessado 2020 Ago 12]. Disponível em: https://tinyurl.com/y38ekh2g

28. Companhia Nacional de Abastecimento (Conab). Últimas Notícias [Internet]. Brasília: Conab; 2020 [acessado 2020 Abr 6]. Disponível em: https://tinyurl.com/ y5onvmlz

29. Ministério Público Federal (MPF). Ação Civil Pública-Processo $n^{\circ}$ 1007677-04.2020.4.01.3200 [Internet] Manaus: Procuradoria da República no Estado do Amazonas; 2020 [acessado 2020 Jun 5]. Disponível em: https://tinyurl.com/y678beek

30. Grisa C, Ávila M, Cabral RF. Iniciativas dos governos estaduais na aquisicao de produtos da agricultura $\mathrm{fa}$ miliar em atendimento as demandas publicas. Brasília; 2020.

31. Organização das Nações Unidas para a Alimentação e a Agricultura (FAO). Addressing the impacts of $\mathrm{CO}$ VID-19 in food crises [Internet]. Rome: FAO; 2020 [acessado 2020 Jun 5]. Disponível em: https://tinyurl. $\mathrm{com} / \mathrm{y} 2 \mathrm{jtgw} 2 \mathrm{c}$

32. Power M, Doherty B, Pybus K, Pickett K. How COVID-19 has exposed inequalities in the UK food system: The case of UK food and poverty. Emerald Open Res 2020; 2:11.

33. Organização das Nações Unidas para a Alimentação e a Agricultura (FAO). COVID-19 and smallholder producers' access to markets. Rome: FAO; 2020.

34. Rede de Informação e Ação pelo Direito a Segurança Alimentar. Impacto da Covid-19 na Realização do Direito Humano à Alimentação e à Nutrição Adequadas: Relatório preliminar de monitoramento [Internet]. Brasília; 2020 [acessado 2020 Jun 5]. Disponível em: https://tinyurl.com/yyt9m5yd

35. Organização Internacional do Trabalho (ILO). COVID-19 and the impact on agriculture and food security [Internet]. Geneva: ILO; 2020 [acessado 2020 Jun 5]. Disponível em: https://tinyurl.com/y56qbbd8

36. Núcleo de Informação e Coordenação do Ponto BR. Survey on the Use of Information and Communication Technologies in Brazilian Households [Internet]. São Paulo: Comitê Gestor da Internet no Brasil; 2019 [acessado 2020 Ago 12]. Disponível em: https:// tinyurl.com/y38nl8gy

37. Brasil. Projeto de Lei $n^{\circ} 735$, de 2020. Dispõe sobre medidas emergenciais de amparo aos agricultores familiares do Brasil para mitigar os impactos socioeconômicos da Covid-19; altera as Leis no ${ }^{\circ}$ 13.340, de 28 de setembro de 2016, e 13.606, de 9 de janeiro de 2018; e dá outras providências (Lei Assis Carvalho). Diário Oficial da União; 2020.

38. DataFavela. Pandemia na favela: a realidade de $14 \mathrm{mi}$ thões de favelados no combate ao novo coronavírus. Rio de Janeiro; 2020

39. Campanha Nacional pelo Direito à Educação (CNDE). Guia Covid-19 Alimentação escolar [Internet]. Brasília: CNDE; 2020. [acessado 2020 Ago 12]. Disponível em: https://tinyurl.com/y5thkbcz 
40. Rossetti FX, Silva MV, Winnie LWY. O Programa Nacional de Alimentação Escolar (PNAE) e o desafio da aquisição de alimentos regionais e saudáveis. Segurança Aliment Nutr 2016; 23(2):912.

41. Programa Alimentar Mundial. Modalidades de compras públicas de alimentos da agricultura familiar no Brasil. Brasília; 2019.

42. Instituto de Pesquisa Econômica Aplicada (IPEA). Evitando a pandemia da pobreza: possibilidades para o Programa Bolsa Família e para o Cadastro Único em resposta à Covid-19 [Internet]. Brasília: IPEA; 2020 [acessado 2020 Ago 13]. Disponível em: https:// tinyurl.com/y6f513xu

43. Siche R. What is the impact of COVID-19 disease on agriculture? Sci Agropecu 2020; 11(1):3-9.

44. Brasil. Medida Provisória ${ }^{\circ}$ 942, de 2 de abril de 2020. Abre crédito extraordinário, em favor da Presidência da República e dos Ministérios da Educação, da Justiça e Segurança Pública, e da Mulher, da Família e dos Direitos Humanos, no valor de R\$ 639.034.512,00, para os fins que especifica. Diário Oficial da União; 2020.

45. Vasconcelos FAG. Combate à fome no Brasil: Uma análise histórica de Vargas a Lula. Rev Nutr 2005; 18(4):439-457.

46. Costa e Silva VL, Turci SRB, Oliveira APN, Richter AP. Can the risk in public-private partnerships be classified? Cad Sau Publ 2017; 33(S3):e00086316.

47. Meireles M. Operação investiga contrato de $R \$ 12,7$ milhões para entrega de cestas básicas a famílias de baixa renda na pandemia pelo governo [Internet]. G1; 2020 [acessado 2020 Ago 17]. Disponível em: https:// tinyurl.com/yy247btq

48. Bernardino G. MPT cobra que governo de Alagoas use Fecoep para fornecer cestas básicas [Internet]. Gazeta Web; 2020 [acessado 2020 Ago 17]. Disponível em: https://tinyurl.com/yy8hcxzo

49. Brasil. Articulação Nacional de Agroecologia (ANA). Nota Técnica $n^{\circ}$ 5/2020. Complementação à Nota Técnica $n^{\circ}$ 3/2020 [Internet]. Brasília: ANA; 2020 [acessado 2020 Ago 13]. Disponível em: https://tinyurl.com/ y4y2jwmw

50. Brasil. Lei $\mathrm{n}^{\circ} 11.346$, de 15 de setembro de 2006. Cria o Sistema Nacional de Segurança Alimentar e Nutricional - SISAN com vistas em assegurar o direito humano à alimentação adequada e dá outras providências. Diário Oficial da União; 2006.

Artigo apresentado em 04/09/2020

Aprovado em 11/09/2020

Versão final apresentda em 13/09/2020 